\title{
Prognostic Value of CD44 Variant exon 6 Expression in Non- Small Cell Lung Cancer: a Meta-analysis
}

\author{
Shuang Zhao ${ }^{1 \&}$, Jin-Lan He ${ }^{2 \&}$, Zhi-Xin Qiu ${ }^{1}$, Nian-Yong Chen², Zhuang Luo ${ }^{1,3}$, \\ Bo-Jiang Chen ${ }^{1}$, Wei-Min Li ${ }^{1 *}$
}

\begin{abstract}
Background: CD44v6 (CD44 variant exon 6) is the chief CD44 variant isoform regulating tumor invasion, progression, and metastasis. The prognostic value of CD44v6 expression in non small cell lung cancer (NSCLC) has been evaluated in many studies, but the results have remained controversial. Thus, we performed a metaanalysis of currently available studies to investigate the prognostic value of CD44v6 expression in NSCLC patients and the relationship between the expression of CD44v6 and clinicopathological features. Materials and Methods: Two independent reviewers searched the relevant literature in Pubmed, Medline and Embase from 1946 to January 2014. Overall survival (OS) and various clinicopathological features were collected from included studies. This meta-analysis was accomplished using STATA 12.0 and Revman 5.2 software. Pooled hazard ratios (HRs) with $95 \%$ confidence intervals $(95 \%$ CIs) were calculated to estimate the effects. Results: A total of 921 NSCLC patients from ten studies met the inclusion criteria. The results showed that CD44v6 high expression was a prognostic factor for poor survival $(\mathrm{HR}=1.91,95 \% \mathrm{CI}=1.12-3.26, p<0.05)$. With respect to clinicopathological features, CD44v6 high expression was related to histopathologic type (squamous cell carcinoma versus adenocarcinoma: $\mathrm{OR}=2.72,95 \% \mathrm{CI}=1.38-5.38, p=0.004)$, and lymph node metastasis $(\mathrm{OR}=3.02$, 95\% CI=1.93-4.72, $p<0.00001)$. Conclusions: Our results suggested CD44v6 high expression as a poor prognostic factor for NSCLC, and CD44v6 expression is associated with lymph node metastasis and histopathologic type. Therefore, CD44v6 expression can be used as a novel prognostic marker in NSCLC cases.
\end{abstract}

Keywords: CD44 variant exon 6 - non-small cell lung cancer - overall survival - meta-analysis

Asian Pac J Cancer Prev, 15 (16), 6761-6766

\section{Introduction}

Lung cancer is the most common malignancy and the leading cause of cancer-related death in men and women worldwide, and non small cell lung cancer (NSCLC) accounts for about $85 \%$ of all lung cancer. Despite the improved surgical techniques and advanced therapeutic regimens, its 5-year overall survival (OS) is still only about $17 \%$ (Siegel et al., 2013). Surgery is thought the best treatment option, but only about $20-25 \%$ of tumours are suitable for potentially curative resection, and most patients have already lost the chance for curative surgery when diagnosed. Thus, there is a great need for identifying prognostic markers to help with early diagnosis and OS for NSCLC.

CD44 is a multi-functional and multi-structural transmembrane glycoprotein, and it was initially characterized as a receptor for hyaluronan and lymphocytehoming receptor (Naor et al., 1997). CD44 plays an important role in prognosis of many cancers, such as bile duct cancer (Kunlabut et al., 2012), breast cancer
(Kaufmann et al., 1995), colorectal cancer (Fan et al., 2012) and so on. CD44 can be expressed as isoforms generated by the alternative splicing of variant exons (Mackay et al., 1994). Specific CD44 isoforms are the surface adhesion molecules, which have been shown to be associated with metastasis. In the early 1990s, it was found that the CD44 variant exon 6 (CD44v6) was the chief variant isoform to regulate tumor invasion, progression, and metastasis in rat carcinoma cells (Gunthert et al., 1991). Meanwhile, CD44v6 is also expressed in both fetal and mature lung, being associated with epithelial stem cells (Jijiwa et al., 2011). Nowadays, several studies have demonstrated that CD44v6 was correlated with the prognosis of ovarian cancer (Shi et al., 2013), colorectal cancer (Wielenga et al., 1993), gastric carcinoma (Mayer et al., 1993), osteosarcoma (Deng et al., 2013), breast cancer (Kaufmann et al., 1995), bladder cancer (Omran et al., 2012) and liver cancer (Coradini et al., 2004). What's more, it has been reported that CD44v6 can contribute to both PI3K/Akt and MAPK activation, which can regulate the extracellular matrix, promote cell motility,

${ }^{1}$ Department of Respiratory Medicine, ${ }^{2}$ Department of Radiation Oncology, State Key Laboratory of Biotherapy and Cancer Center, West China Hospital, Sichuan University, Chengdu, ${ }^{3}$ Department of Respiratory Medicine, First Affiliated Hospital of Kunming Medical university, Kunming, China ${ }^{\circledR}$ Equal contributors *For correspondence: weimin003@163.com 
and suppress cancer apoptosis (Marhaba et al., 2005; Jung et al., 2011).

The prognostic value of CD44v6 immunohistochemical expression in patients with NSCLC has been evaluated in many studies, but the results are controversial. Thus, we performed a meta-analysis of currently available studies to investigate the prognostic value of CD44v6 expression in NSCLC and the relationship between the expression of CD44v6 and clinicopathological features.

\section{Materials and Methods}

\section{Literature search}

We performed a comprehensive literature search of the following databases: Pubmed, Medline and Embase from from 1946 to January 2014, and the keywords were ("CD44v6" or "CD44 variant exon 6" or "CD44 variant 6"or "CD44 variant isoform v6") and ("lung neoplasm" or "lung cancer" or "lung carcinoma" or "non small cell lung cancer"). Titles and abstracts were evaluated to identify the relevant literature, and then full texts were carefully read.

\section{Selection criteria}

1.Diagnosis of NSCLC was proven by histopathological methods and complied with the diagnosis criteria of the World Health Organization (WHO). 2. Expression of CD44v6 was detected by immunohistochemistry (IHC). 3 . The studies offered sufficient data for estimating hazard ratios (HRs) and their 95\% confidence intervals (95\%CIs). 4. The samples of the studies for detecting CD44v6 expression were surgically resected lung cancer tissues, rather than body fluids such as peritoneal fluid, serum and sputum. 5. The literature should be published with full texts. 6 . Articles published as reviews, conference abstracts or comments were excluded.

There was no limitation on language or the minimum number of patients. When the same author reported results performed in the same population, only the most recent or the most complete report was included in the analysis.

\section{Data extraction}

Two reviewers (Z.S and H.J.L) searched the articles, extracted data and checked all potentially relevant literature independently. All disagreements in data extraction were settled by consensus, referring back to the original article and those could not be settled were resolved by a third reviewer (Q.Z.X) through discussion. The following data were collected from each article: first author, year of publication, country, number of patients, CD44v6 high/ low expression cases, laboratory methodology, cut-off score, follow-up period, information about neo-adjuvant therapy, HR with 95\%CI and clinicopathological features such as tumor differentiation, histological type, lymph node metastasis, tumor stages.

From some published researches, HR and 95\%CI could be directly obtained by using survival analysis. For articles that didn't provide HR and 95\%CI directly , two reviewers (Z.S and H.J.L) independently digitized and extracted the data through the Kaplan-Meier curves by using GetData Graph Digitizer 2.24 (http://getdata-graphdigitizer.com) and then extracted data were utilized to reconstruct the HR and its variance (GraphPad Software, Inc, La Jolla, CA, USA).

\section{Statistical analysis}

STATA 12.0 software (STATA Corp., College Station, TX) and Revman 5.2 software (Cochrane Collaboration, Copenhagen) were used to perform statistical analysis (Higgins et al., 2011). The association between CD44v6 expression and survival were described as HR, and the strength of association between CD44v6 and clinicopathological features were expressed as odds ratios (OR). High expression of CD44v6 indicated poor prognosis in patients with NSCLC if $\mathrm{HR}>1$ with the 95\% CI did not overlap 1. Cochrane's Q test (Chi-squared test; Chi2) and inconsistency (I2) statistics were performed to assess the heterogeneity (Lau et al., 1997; Higgins et al., 2002). Random-effect model was used to calculate pooled estimates when the P value of heterogeneity was less than 0.05. Otherwise, a fixed-effect model was used. Begg rank correlation and Egger linear regression tests were designed for assessing risk of publication bias (Macaskill et al., 2001). P value $<0.05$ was considered as statistically significant. Additionally, sensitivity analysis was used to assess the stability of the results.

\section{Results}

\section{Description of search results}

196 studies were retrieved initially using the above search strategy. Then according to the selection criteria, 152 studies were excluded through reading titles and abstracts. The remaining 45 candidate studies were further evaluated by reading the full-texts. 16 studies were excluded because they were irrelevant studies on CD44v6 expression in lung cancer, 3 studies were excluded because the laboratory methodology were not immunohistochemistry, and 16 studies were excluded because they were without survival statistics or could not offer sufficient data for estimating HR and 95\%CI. Eventually, a total of ten independent studies were included in the present meta-analysis (six in English and four in Chinese) (Figure 1).

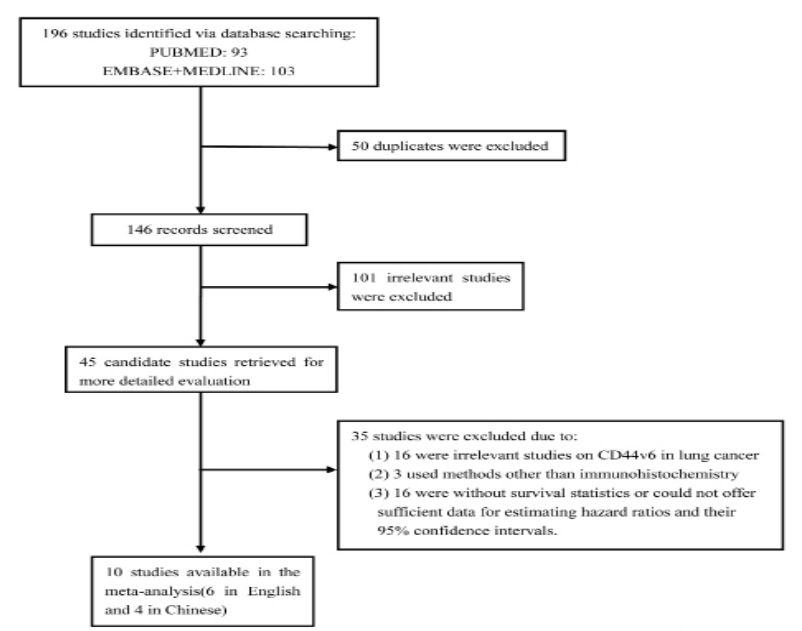

Figure 1. Flow Chart of the Literture Search and Selection of Included Studies 
Characteristics of eligible studies

The main characteristics of the ten studies eligible for the metaanalysis are shown in Table 1 . The total number of patients included was 921 ranging from 34 to 190 patients per study (Hirata et al., 1998; Fukuse et al., 1999; Nguyen et al., 2000; Ramasami et al., 2000; Mei et al., 2004; Zhang et al., 2004; Wu et al., 2005; Weng et al., 2008; Situ et al., 2010; Sun et al, 2013). Among the ten studies, two studies were from Europe and eight were from Asia. The mean follow-up period for the studies was 54.9 months ranging from 24 to 72 months per study. The median percentage of patients with CD44v6 high expression was $46.99 \%$ ranging $28.33 \%$ to $69.36 \%$ per study. Histopathologic types were reported in ten studies and lymph node metastases were reported in seven studies. The TNM stages were reported in seven studies and tumor grade were reported in four studies. Because none of these studies have analyzed the relationship between the CD44v6 expression and disease free survival rate (PFS) in NSCLC patients, we could have only gathered the OS information and conducted the meta-analysis about the association between the expression level of CD44v6 and OS. Seven of the ten included studies identified CD44v6 high expression as a significant poor prognostic factor, whereas the other three studies reported that CD44v6 high expression was a good prognostic factor or non-significant association with prognosis.

\section{CD44v6 expression and overall survival of NSCLC patients}

Ten studies had sufficient data for estimating HR and 95\%CI, including 921 patients. As shown in Figure 2, CD44v6 high expression was a prognostic factor for poor survival in NSCLC patients $(\mathrm{HR}=1.91,95 \% \mathrm{CI}=1.12-3.26, \mathrm{P}<0.05)$. The random-effects model was used because a significant heterogeneity was observed among these studies $\left(p=0.000, \mathrm{I}^{2}=82.4 \%\right)$. Our results indicated that CD44v6 was an independent prognostic factor in patients with NSCLC.

\section{CD44v6 expression and clinicopathological features}

As shown in Table 2. Six studies assessed the relationship between CD44v6 expression and lymph node metastasis, including 471 patients. Eight studies had sufficient data for assessing the relationship between CD44v6 expression and histopathologic type, including 645 patients. The results suggested that the CD44v6 high expression was associated with clinicopathological features such as histopathologic type (squamous cell carcinoma versus adenocarcinoma: pooled $\mathrm{OR}=3.02,95 \% \mathrm{CI}=1.93-4.72, p<0.00001$, Fixed-effect) and lymph node metastasis (pooled OR=2.72, 95\%CI: 1.38-5.38, $p=0.004$, Random-effect). However, no significant correlations were found between $\mathrm{CD} 44 \mathrm{v} 6$ expression and gender $(\mathrm{OR}=1.3,95 \% \mathrm{CI}=0.73$ -

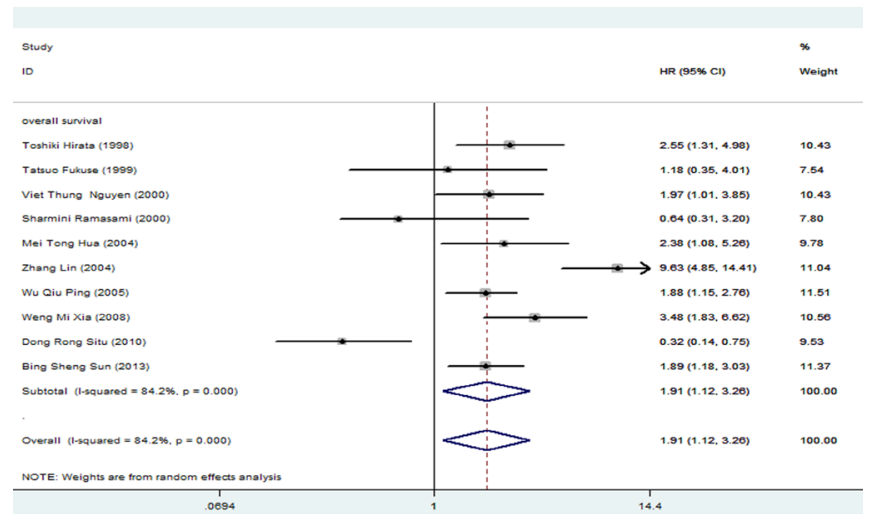

Figure 2. Forest Plot Showing the Combined Relative HR from the Random-Effect Model for Overrall Survivall

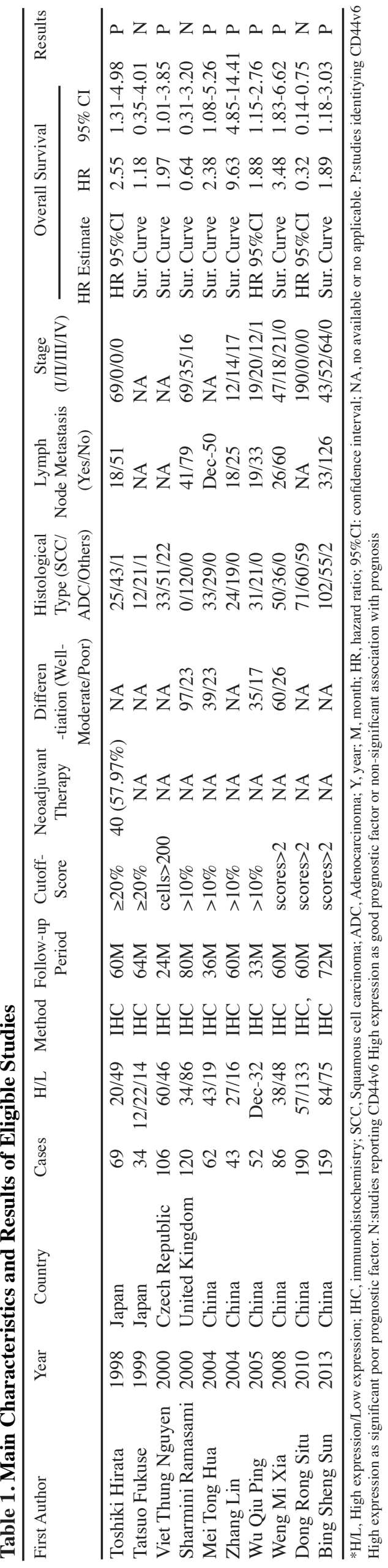

Asian Pacific Journal of Cancer Prevention, Vol 15, 2014 
Table 2. Meta-Analysis Assessing the Association between CD44v6 high Expression and Clinicopathological Features

\begin{tabular}{|c|c|c|c|c|c|c|c|c|c|}
\hline \multirow[t]{2}{*}{ Clinicalpathological features } & \multirow[t]{2}{*}{ Studies (n) } & \multirow{2}{*}{$\begin{array}{l}\text { Effect } \\
\text { model }\end{array}$} & \multicolumn{3}{|c|}{ Pooled Data } & \multirow[b]{2}{*}{$p$ value } & \multicolumn{3}{|c|}{ Test for Heterogeneity } \\
\hline & & & Cases & OR & $95 \% \mathrm{CI}$ & & $\mathrm{Chi}^{2}$ & $P$-value & $\mathrm{I}^{2}(\%)$ \\
\hline$\overline{\text { Gender (male/female) }}$ & 2 & Fixed & 221 & 1.3 & $0.73-2.30$ & 0.37 & 0.6 & 0.44 & 0 \\
\hline Histological Type (SCC/ADC) & 8 & Random & 645 & 2.72 & $1.38-5.38$ & 0.004 & 23.76 & 0.001 & 71 \\
\hline Differentiation (well-moderate/poor) & or) 3 & Random & 200 & 2.11 & $0.63-7.11$ & 0.23 & 5.57 & 0.06 & 64 \\
\hline Lymph Node Metastasis & 6 & Fixed & 471 & 3.02 & $1.93-4.72$ & $<0.00001$ & 7.52 & 0.18 & 34 \\
\hline TNM Stage (I-II/III-IV) & 4 & Random & 359 & 1.02 & $0.23-4.56$ & 0.98 & 20.19 & 0.0002 & 85 \\
\hline
\end{tabular}

*No: number; SCC: Squamous cell carcinoma; ADC: Adenocarcinoma

Table 3. Results of Subgroup Analyses

\begin{tabular}{|c|c|c|c|c|c|}
\hline \multirow[t]{2}{*}{ Gategories } & \multirow{2}{*}{$\begin{array}{c}\text { Studies } \\
\text { (n) }\end{array}$} & \multicolumn{2}{|c|}{ Heterogeneity } & \multirow{2}{*}{$\begin{array}{l}\text { Effect } \\
\text { model }\end{array}$} & \multirow{2}{*}{$\begin{array}{c}\text { HR } \\
(95 \% \mathrm{CI})\end{array}$} \\
\hline & & $\mathrm{I}^{2}(\%)$ & $p$ values & & \\
\hline \multicolumn{6}{|l|}{ Ethnicity } \\
\hline Asian & 8 & 86.5 & 0 & Random & $2.11(1.14-3.90)$ \\
\hline European & 2 & 62.7 & 0.101 & Fixed & $1.49(0.83-2.67)$ \\
\hline \multicolumn{6}{|l|}{ Year of pubilish } \\
\hline Before 2000 & 4 & 34.6 & 0.204 & Fixed & $1.78(1.18-2.69)$ \\
\hline After 2000 & 6 & 90.1 & 0 & Random & $2.18(1.02-4.68)$ \\
\hline \multicolumn{6}{|l|}{ Cut off scores } \\
\hline$>10 \%$ & 4 & 89.7 & 0 & Random & $2.46(0.88-6.88)$ \\
\hline$\geq 20 \%$ & 2 & 15.3 & 0.277 & Fixed & $2.13(1.19-3.83)$ \\
\hline Scores $>2$ & 3 & 90.1 & 0 & Random & $1.33(0.41-4.28)$ \\
\hline \multicolumn{6}{|c|}{ Follow-up period } \\
\hline$<60$ month & 3 & 0 & 0.877 & Fixed & $1.98(1.42-2.76)$ \\
\hline$\geq 60$ month & 7 & 89.2 & 0 & Random & $1.79(0.79-4.05)$ \\
\hline
\end{tabular}

2.30, $p=0.37$, Fixed-effect), tumor differentiation (OR=2.11,95\%CI=0.63-7.11, $p=0.23$, Random-effect $)$ or pathological stage $(\mathrm{OR}=1.02,95 \% \mathrm{CI}=0.23-4.56, p=0.98$, Random-effect).

\section{Subgroup analysis}

We performed subgroup analysis in order to further explain the results of OS. Ethnicity, year of publication , cut off scores and follow-up period were included as factors in subgroup analysis Table 3). After stratifying by ethnicity, the pooled HRs of Asians and Europeans were $2.11(95 \% \mathrm{CI}=1.14-3.90$, Random-effect) and 1.49 (95\%CI: 0.83-2.67; Fixed-effect), respectively. When stratified by year of publish, a significant association between CD44v6 high expression and prognosis in NSCLC patients was found among the studies published after 2000 (HR=2.18,95\%CI=1.02-4.68, Random-effect), whilst among the studies published before 2000, the statistical significance still existed $(\mathrm{HR}=1.78,95 \% \mathrm{CI}=$ 1.18-2.69; Fixed-effect). In the subgroup analysis based on cut off scores, the pooled HR for scores $\geq 20 \%$ was 2.13 (95\% CI=1.19-3.83, Fixed-effect), the pooled HR for scores $\geq 10 \%$ was 2.46 (95\% CI=0.88-6.88, Randomeffect) and the pooled HR for scores $\geq 2$ was 1.33 (95\%CI=0.41-4.28, Random-effect). Subgroup analysis stratified according to the follow-up period revealed that, in the studies of shorter follow-up period ( $<60$ month), the pooled HR was 1.98 (95\%CI: 1.42-2.76, Fixed-effect), while in studies with longer follow-up period ( $>60$ month) there was no statistical significance $(\mathrm{HR}=1.79,95 \% \mathrm{CI}$ : 0.79-4.05, Random -effect).

\section{Publication bias}

Publication bias was detected in this meta-analysis
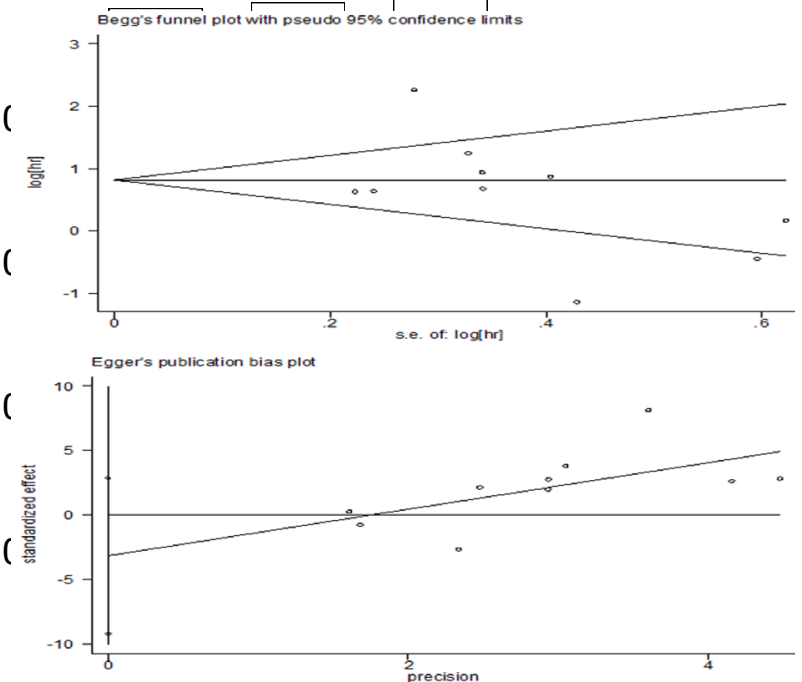

Figure 3. Begg's and Egger's Funnel Plot to Detect Publication Bias

by using the Begg rank correlation and Egger linear regression tests (Figure 3). The association between CD44v6 expression and the OS in NSCLC patients had no significant publication bias existed (Begg's $P$ value $=0.180$, Egger's $\mathrm{P}$ value $=0.886)($ All $p>0.05)$. Thus, our results were statistically reliable.

\section{Discussion}

This meta-analysis is the first study to estimate the association between CD44v6 expression and the overall survival in NSCLC patients. Based on our results, CD44v6 high expression is a prognostic factor for poor survival in NSCLC patients. With respect to clinicopathological features, the present results indicate that CD44v6 high expression was significantly associated with histopathologic type and lymph node metastasis. Nevertheless, there were no statistically significant differences between CD44v6 expression and gender, tumor differentiation or pathological stage.

CD44 variant isoforms, especially CD44v6, have been identified as protein markers for metastatic behavior in many malignant tumors. Fan et al. performed a metaanalysis and demonstrated CD44v6 positive cells were significantly associated with poor overall survival in patients with colorectal cancer $(\mathrm{OR}=0.36, p=0.02)$ (Fan et al., 2012). Study by Chen suggested that CD44v6/v7 expression might be a prognostic indicator in patients with acute promyelocytic leukemia (Chen et al., 2012). Spaford et al. reported that CD44v6 high expression was 
consistent with longer survival in laryngeal squamous cell carcinoma patients. In lung cancer, the present studies showed CD44v6 may play an important role in lung carcinoma histogenesis and in determining the fate of neoplastic differentiation (Spaford et al., 1996). Afify et al. found CD44v6 expression was more significantly associated with tumor size in lung cancer (Afify et al., 2011). Study by Sun et al. elucidated that CD44v6 might be correlated with the histogenesis of NSCLC, and decreased CD44v6 expression was an adverse prognostic indicator for squamous cell carcinoma rather than adenocarcinoma or bronchioalveolar carcinoma (Sun et al., 2013). Another study found the positive expression rate of CD44v6 was significantly lower in the NSCLC tissue without lymphatic metastasis, which was accordant with our results, and detection of CD44v6 expression could be used to judge the lymphatic metastasis in NSCLC (Su et al., 2014). The mechanism of CD44v6 promoting the metastasis of cancer may be attributed to its interactions with various components of the extracellular matrix, and its involvement in cell adhesion and critical signaling pathways. Jung et al. found the CD44v6 could activate the MAPK and the PI3K/Akt pathways, and then the two pathways could activate anti-apoptotic proteins and inactivate pro-apoptotic proteins (Jung et al., 2004). Their study demonstrated that CD44v6 could essentially contribute to tumor progression. Therefore, CD44v6 expression can be used as a novel prognostic marker in lung cancer.

A significant heterogeneity was observed among these included studies in this meta-analysis, and through the subgroup analysis, we can see the heterogeneity may be produced by the cut-off scores, ethnicity, year of publication and follow-up period. We also noticed that some studies had examined CD44v6 expression in NSCLC patients using methods other than immunohistochemistry (Miyoshi et al., 1997; Lee et al., 2005; Liu et al., 2005). Using reverse transcription PCR, Lee found that CD44v6 low expression was correlated to short disease-free interval and overall survival in NSCLC (Lee et al., 2005), this result was conflict with our meta-analysis. But the study by Miyoshi et al. was consistent with our results, they examined the expression of CD44 variant forms in 31 NSCLC patients and 8 normal lung tissue samples by reverse transcription-PCR, they found the expression of CD44v6 was particularly associated with lymph node metastasis in NSCLC (Miyoshi et al., 1997). Thence, in order to establish a sensitive and specific detecting method, further studies with a suitable laboratory methodology and unified cut-off scores are needed.

Although we performed a comprehensive analysis of the association between CD44v6 expression and NSCLC patients, certain limitations of this study should be considered. First of all, no RCTs had been found, and the number of included studies was relatively small with only about 921 cases. Secondly, due to the lack of original information for each study, the reconstructed HR from the survival curves inevitably reduced the accuracy and enhanced the uncertainty of the estimates. Thirdly, though IHC was used for evaluating CD44v6 expression, the types or the dilutions of primary antibody were not the same in all of the included studies. Because different antibody concentration may lead to different sensitivity of IHC, a potential bias might exist. Fourthly, our meta-analysis data was insufficient on age, smoking status, tumor size and other factors, which might result in confounding bias. Fifthly, though our results of publication bias showed no significance, a possible bias could not be excluded because some other relevant published or unpublished studies may not be included due to their absence in our selected databases. So the results should be interpreted cautiously.

In conclusion, despite the limitations mentioned above, our meta-analysis of the studies suggested CD44v6 high expression might be a poor prognostic factor for patients with NSCLC, and CD44v6 expression was related to lymph node metastasis and histopathologic type. Nevertheless, since the reported associations were diverse, these findings need to be interpreted with caution when used in clinical practice. A large cohort study is needed to further demonstrate the association between CD44v6 and the survival of patients with NSCLC.

\section{Acknowledgements}

This research is supported by grants from the Nature Science Foundation of China (81241068, 81372504).

\section{References}

Afify AM, Tate S, Durbin-Johnson B, et al (2011). Expression of CD44s and CD44v6 in lung cancer and their correlation with prognostic factors. Int J Biol Markers, 26, 50-7.

Coradini D, Zorzet S, Rossin R, et al (2004). Inhibition of hepatocellular carcinomas in vitro and hepatic metastases in vivo in mice by the histone deacetylase inhibitor HA-But. Clin Cancer Res, 10, 4822-30.

Chen P, Huang HF, Lu R, et al (2012). Prognostic significance of CD44v6/v7 in acute promyelocytic leukemia. Asian Pac J Cancer Prev, 13, 3791-4.

Deng Z, Niu G, Cai L, et al (2013). The prognostic significance of CD44V6, CDH11, and $\beta$-Catenin expression in patients with osteosarcoma. Biomed Res Int, 2013, 496193.

Fan CW, Wen L, Qiang ZD, et al (2012). Prognostic significance of relevant markers of cancer stem cells in colorectal cancer-a meta analysis. Hepatogastroenterology, 59, 1421-7.

Fukuse T, Hirata T, Naiki H, et al (1999). Expression of proliferating cell nuclear antigen and CD44 variant isoforms in the primary and metastatic sites of nonsmall cell lung carcinoma with intrapulmonary metastases. Cancer, 86, 1174-81.

Gunthert U, Hofmann M, Rudy W, et al (1991). A new variant of glycoprotein CD44 confers metastatic potential to rat carcinoma cells. Cell, $\mathbf{6 5}, 13-24$.

Higgins JP, Thompson SG (2002). Quantifying heterogeneity in a meta-analysis. Stat Med, 21, 1539-58.

Higgins JPT, Green S (2011). Cochrane Handbook for Systematic Reviews of Interventions Version 5.1.0. The Cochrane Collaboration, 2011. Available:www.cochranehandbook.org. [updated March 2011]

Hirata T, Fukuse T, Naiki H, et al (1998). Expression of CD44 variant exon 6 in stage I non-small cell lung carcinoma as a prognostic Factor. Cancer Res, 58, 1108-10.

Jijiwa M, Demir H, Gupta S, et al (2011). CD44v6 regulates growth of brain tumor stem cells partially through the AKTmediated pathway. Plos One, 6, 24217. 


\section{Shuang Zhao et al}

Jung T, Gross W, Zoller M (2011). CD44v6 coordinates tumor matrix-triggered motility and apoptosis resistance. $\mathrm{J}$ Biol Chem, 286, 15862-74.

Kunlabut K, Vaeteewoottacharn K, Wongkham C, et al (2012). Aberrant expression of CD44 in bile duct cancer correlates with poor prognosis. Asian Pac J Cancer Prev, 13, 95-9.

Kaufmann M, Heider KH, Sinn HP, et al (1995). CD44 variant exon epitopes in primary breast cancer and length of survival. Lancet, 345, 615-9.

Lau J, Ioannidis JP, Schmid CH (1997). Quantitative synthesis in systematic reviews. Ann Intern Med, 127, 820-6.

Lee LN, Kuo SH, Lee YC, et al (2005). CD44 splicing pattern is associated with disease progression in pulmonary adenocarcinoma. J Formos Med Assoc, 104, 541-8.

Liu YJ, Yan PS, Li J, et al (2005). Expression and significance of CD44s, CD44v6, and nm23 mRNA in human cancer. World J Gastroenterol, 11, 6601-6.

Mayer B, Jauch KW, Gunthert U, et al (1993). De-novo expression of CD44 and survival in gastric cancer. Lancet, 342, 1019-22.

Mackay CR, Terpe HJ, Stauder R, et al (1994). Expression and modulation of CD44 variant isoforms in humans. J Cell Biol, 124, 71-82.

Macaskill P, Walter SD, Irwig L (2001). A comparison of methods to detect publication bias in meta-analysis. Stat Med, 20, 641-54.

Marhaba R, Bourouba M, Zoller M (2005). CD44v6 promotes proliferation by persisting activation of MAP kinases. Cell Signal, 17, 961-73.

Miyoshi T, Kondo K, Hino N, et al (1997). The expression of the CD44 variant exon 6 is associated with lymph node metastasis in non-small cell lung cancer. Clin Cancer Res, 3, 1289-97.

Mei TH, Zhang GR, Ma Y (2004). The expression of vascular endothelial growth factor, CD44v6 in non-small cell lung carcinoma. Zhonghua Wai Ke Za Zhi, 42, 647-50.

Naor D, Sionov RV, Ish-Shalom D (1997). CD44: structure, function, and association with the malignant process. $A d v$ Cancer Res, 71, 241-319.

Nguyen VN, Mirejovsky T, Melinova L, et al (2000). CD44 and its v6 spliced variant in lung carcinomas: relation to NCAM, CEA, EMA and UP1 and prognostic significance. Neoplasma, 47, 400-8.

Omran OM, Ata HS (2012). CD44s and CD44v6 in diagnosis and prognosis of human bladder cancer. Ultrastruct Pathol, 36, 145-52.

Ramasami S, Kerr KM, Chapman AD, et al (2000). Expression of CD44v6 but not E-cadherin or beta-catenin influences prognosis in primary pulmonary adenocarcinoma. J Pathol, 192, 427-32.

Sun BS, Li Y, Zhang ZF, et al (2013). Osteopontin combined with CD44v6, a novel prognostic biomarker in non-small cell lung cancer undergoing curative resection. Ann Thorac Surg, 96, 1943-51.

Su CY, Li YS, Han Y, et al (2014). Correlation between expression of cell adhesion molecules CD44v6 and E-cadherin and lymphatic metastasis in non- small cell lung cancer. Asian Pac J Cancer Prev, 15, 2221-4.

Situ D, Long H, Lin P, et al (2010). Expression and prognostic relevance of CD44v6 in stage I non-small cell lung carcinoma. J Cancer Res, 136, 1213-9.

Shi J, Zhou Z, Di W, et al (2013). Correlation of CD44v6 expression with ovarian cancer progression and recurrence. BMC Cancer, 13, 182.

Spaford MF, Koeppe J, Pan Z, et al (1996). Correlation of tumor markers p53, bcl-2, CD34, CD44H, CD44v6, and Ki-67 with survival and metastasis in laryngeal squamous cell carcinoma. Arch Otolaryngol Head Neck Surg, 122, 627-32. Siegel R, Naishadham D, Jemal A (2013). Cancer statistics, 2013. CA Cancer J Clin, 63, 11-30.

Weng MX, Wu CH, Yang XP(2008). Expression and significance of E-cadherin, CD44v6, and proliferating cell nuclear antigen in non-small cell lung cancer. Ai Zheng, 27, 191-5.

Wu Q, Jiang Y, Min J, et al (2005). Expression of CD44v6 and its prognostic significance in non-small cell lung cancer. Zhongguo Fei Ai Za Zhi, 8, 215-8.

Wielenga VJ, Heider KH, Offerhaus GJ, et al (1993). Expression of CD44 variant proteins in human colorectal cancer is related to tumor progression. Cancer Res, 53, 4754-6.

Zhang L, Meng L, Wang L, et al (2004). The clinical significance of detection of vascular endothelial growth factor and CD44v6 expression in human non-small cell lung cancer. Zhongguo Fei Ai Za Zhi, 7, 427-30. 